\title{
Hybrid Magnetic Suspension Actuator for Precision Motion Control
}

\author{
Dengfeng $\mathrm{Li}^{1}$ and Hector Gutierrez ${ }^{2}$ \\ ${ }^{1}$ Laser Photonics, LLC \\ ${ }^{2}$ Florida Institute of Technology \\ USA
}

\section{Introduction}

The blooming development of semiconductor fabrication technologies, such as those used for manufacturing of micro electromechanical systems, has increased the interest to develop actuators that can provide micro scale or nano scale motion, with large range of travel range and fast dynamic response. For these applications, the main candidate technologies are magnetic suspension actuation (MSA) and piezoelectric actuation (PZT).

Direct piezoelectric actuation (i.e., actuation based on the strain of a piezo stack by the application of voltage or charge) has inherently a very limited range of travel. Although the displacement can be enlarged by mechanical amplification (Robbins, 1991) the range available for direct PZT actuation is at most in the order of a few hundred microns, and the precise control of PZT actuators involves other challenges such as hysteresis and drift. To achieve millimeter-range travel with piezoelectric actuation, ultrasonic motors or inchworm mechanisms have been devised. These devices can achieve excellent positioning performance but are limited in their force and torque load capacity, and have small tracking bandwidth.

MSA technologies are an interesting alternative to PZT in that they can provide substantially larger load capacity, fast response and large range of travel without introducing the complex microscopic phenomena associated with mechanical contact. The MSA devices based on attractive force can be found in works of Trump et al (Trump,1997; Kuo, 2003). All of these are small gap actuators which is not suitable for applications where the actuator is remote from the controlled target. More recently, large gap MSA devices which magnify the magnetic field to overcome the gap limitation can be found in (Lin, 2007) (Craig, 2007) and (Khamesee, 2005). However, it seems that the mass of the controlled object is required to be very small. When the mass and air gap become larger, a potential problem is the steep increase in required control effort (actuator current) may lead to heat dissipation problems and actuator core saturation. A novel hybrid MSA concept which has a great promise to realize large gap while avoiding the need of large currents and actuator saturation is presented in this paper. In the proposed design, a pair of permanent magnets in repulsive configuration is located coaxial with an attractive force electromagnet, as shown in Fig. 1. In this arrangement, zero current is needed to obtain large gap since passive force is used. Controlled attractive forces are used to provide stabilization of the controlled object. 
This push-and-pull strategy is particularly useful when a large mass is to be suspended over a large range of travel, since the nominal gap is mainly dependent on the strength of the permanent magnets.

The dynamics of a MSA actuator are dominated by the nonlinear force-gap-current relationship. The most common controller design approach has been the use of linear control based on the linearization of the nonlinear dynamics around a nominal operation point. Controller performance deteriorates rapidly when the actuator position moves away from the nominal operation point. In (Trump, 1997) and (Ludwick, 1996), feedback linearization is successfully applied to a class of systems that can be described by the controllability canonical form. However, to achieve its ideal performance, feedback linearization requires perfect knowledge of the system's dynamics. In an application with large range of travel, parameter variation presents a considerable disturbance that has to be compensated by the controller. A robust nonlinear compensation scheme that synthesizes feedback linearization and disturbance estimation is presented in (Mittal, 1997). Variable structure control (VSC) is another good choice to provide robustness to external perturbations and model uncertainties. In (Gutierrez, 2005), a modified equivalent control approach is used to describe the dynamics of sliding mode. However, a reaching condition that uses a Lyapunov function approach ensures good transient performances only in systems with a single input since there is single sliding surface (Hung, 1993). In systems with multiple inputs there might be multiple sliding surfaces, and the transient performances on some sliding surfaces are typically unknown which might induce slow response or chattering. To obtain fast response and low chatter in a MSA application with long range of travel, an enhanced quasi-sliding mode control with reaching law is proposed and its performances are compared with the PID control with feedback linearization.

\section{Actuator design and force model parameters identification}

The proposed hybrid MSA is shown in Fig. 1, where two cylinders composed of four NdFeB magnets are placed in coaxial with a round electromagnetic coil. To reduce flux leakage, the permanent magnets are coated with ferrous sleeves. The nominal levitation gap can be modified by changing the length of the permanent magnet stack. The axial force in the HMSA is therefore composed of two components: a repulsive force exerted by the permanent magnets, and an attractive force exerted by the coil.

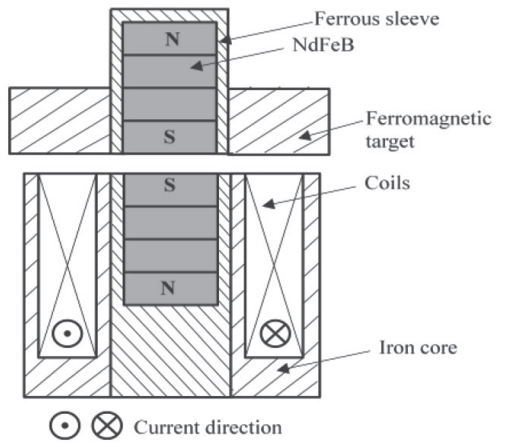

Fig. 1. Hybrid Magnetic Suspension Actuator 
The flux lines shown in Fig. 2 demonstrate that the flux leakage can be neglected due to the high permeability of ferrous sleeve. It's reasonable to assume that both the permanent magnet stack have constant flux. The magnetomotive force of each permanent magnet stack is (Chikazumi,2005):

$$
\mathcal{F}=\frac{B_{r}}{\mu} L
$$

in which $B_{r}$ is the residual induction of the permanent magnet, $L$ is the length of the stack and $\mu$ is permeability of $\mathrm{NdFeB}$. When the values are $\mathrm{B}_{\mathrm{r}}=12000$ Gauss, $\mathrm{L}=31.75 \mathrm{~mm}, \mu=1.15 \mu_{0}$, the magnetomotive force of permanent magnet is 26364 ampere-turn.

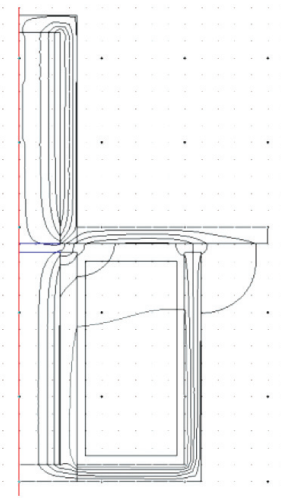

Fig. 2. Flux Lines in Hybrid Magnetic Suspension Actuator

Since the allowed current passing through the coils is designed to be less than $2 \mathrm{~A}$ and the total number of turns is 270 , the NI value of the coil is 540 ampere-turn which is far less than the magnetomotive force of the permanent magnet. This ensures that the moving permanent magnet will not be demagnetized by the field of the coils. The parameters of the proposed HMSA are listed in Table 1.

\begin{tabular}{|l|l|l|l|l|l|l|}
\hline $\begin{array}{c}\text { Diameter } \\
\text { of NdFeB } \\
(\mathrm{mm})\end{array}$ & $\begin{array}{c}\text { Thickness } \\
\text { of NdFeB } \\
(\mathrm{mm})\end{array}$ & $\begin{array}{c}\text { Residual } \\
\text { induction } \\
(\text { Gauss })\end{array}$ & $\begin{array}{c}\text { Thickness } \\
\text { of sleeve } \\
(\mathrm{mm})\end{array}$ & $\begin{array}{c}\text { Length of } \\
\text { Coil } \\
\text { winding } \\
(\mathrm{mm})\end{array}$ & $\begin{array}{c}\text { Coil } \\
\text { Diameter } \\
(\mathrm{mm})\end{array}$ & $\begin{array}{c}\text { Number } \\
\text { of turns }\end{array}$ \\
\hline 9.47 & 7.93 & 12000 & 2.25 & 30.48 & 0.5 & 270 \\
\hline
\end{tabular}

Table 1. Parameters of Hybrid Magnetic Suspension Actuator

In order to calculate the force of permanent magnet, a parametric simulation is performed using Ansoft Maxwell as shown in Fig. 3. Then a zero-current test was performed to characterize the force exerted by the permanent magnets. A one-DOF experimental setup to determine force model parameter is shown in Fig. 4, where a ferromagnetic target is mounted at the end of an aluminum beam supported on a flexure. The HMSA is mounted on a clamp which is attached to the base plate. The gap is sensed by a capacitance gauge with an operating range of 500 to $1500 \mu \mathrm{m}$, and the nominal levitation gap is determined by the relative location of the permanent magnets. The force of permanent magnet is measured 
by a load cell mounted on a clamp. By adjusting the screw, the air gap is gradually increased from 500 to $1000 \mu \mathrm{m}$ without applying current in the coils. The force corresponding to each gap value is recorded and plotted in Fig. 3 to compare with the simulation data. One can see that in the working range of our selected capacitive sensor the force gap relationship can be approximately treated as linear for simplicity.
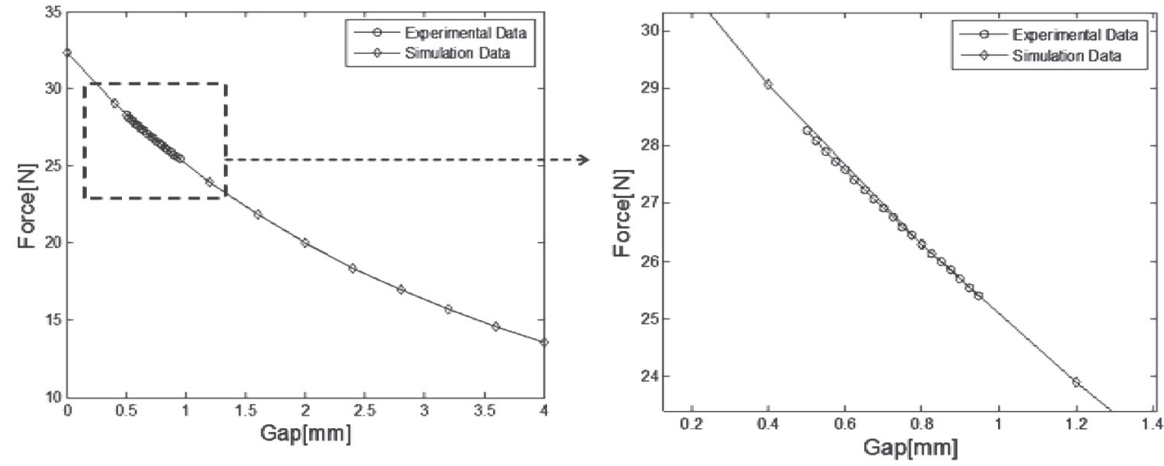

Fig. 3. Experimental and Simulation Repulsive Force Exerted by the Permanent Magnets in the HMSA with zero Current (Left) full view (Right) zoom-in view
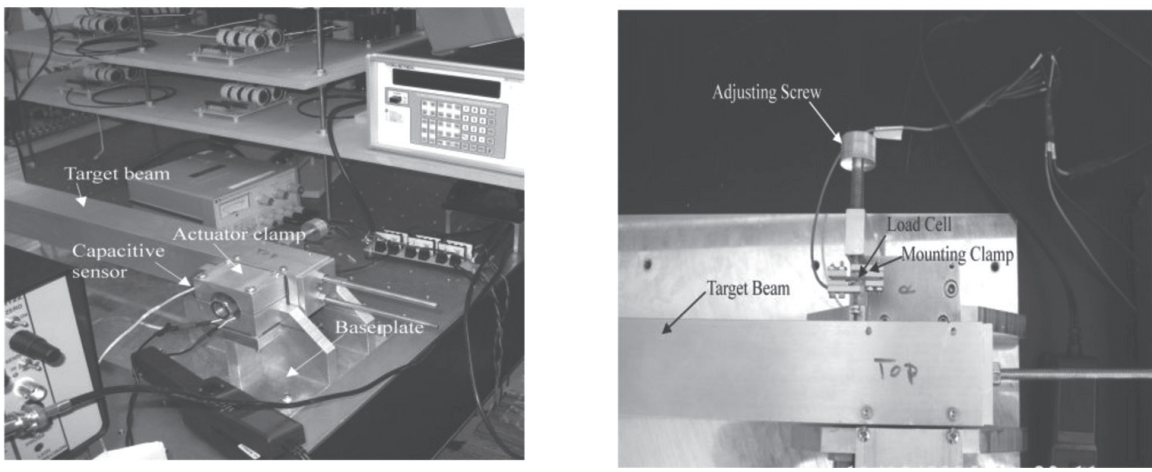

Fig. 4. One DOF Experimental Setup (Left) front view (Right) top view

It is well known that the force produced by attractive-type MSA actuators is proportional to the current squared and inversely proportional to the gap squared. Several methods to find force parameters of attractive-type MSA actuators have been described (Oliveira,1999; Mittal,1997; Lin,1993). This paper takes into account the force of permanent magnet discussed above in the force model of HMSA. Thus, the model for the axial force generated by the HMSA is proposed as:

$$
F=K_{1} g+K_{2}-K_{3} \frac{I^{2}}{\left(g+x_{0}\right)^{2}}
$$

where $g$ is the air gap, $I$ is the coil current , $K_{1}$ and $K_{2}$ are constants related to the force exerted by the permanent magnets, $K_{3}$ and $x_{0}$ are constants related to the electromagnetic 
attractive force. $K_{1}$ and $K_{2}$ were first calculated by optimal polynomial fit using the experimental data shown in Fig. 3. After this, DC currents were applied to the HMSA coil, and the corresponding forces and gaps were measured. To cover the same gap range as used for the zero-current measurements, nine data sets were acquired over nine intervals covering the proposed range. Denoting the measured gap, force and current as $g_{M E A}, F_{M E A}$ and $I_{M E A}$ respectively, the parameter identification problem can be converted to a multidimensional unconstrained nonlinear optimization problem given by:

$$
\min \sum\left(F_{M E A}-\left(K_{1} g_{M E A}+K_{2}-K_{3} \frac{I_{M E A}{ }^{2}}{\left(g_{M E A}+x_{0}\right)^{2}}\right)\right)^{2}
$$

The initial guesses for $K_{1}$ and $K_{2}$ were chosen close as the values calculated from Fig. 3 . The model parameters in (1) were found to be $K_{1}=-6523.7 \mathrm{~N} / \mathrm{m}, K_{2}=29.588 \mathrm{~N}$, $K_{3}=7.0306 \times 10^{-6} \mathrm{Nm}^{2} / A^{2}$, and $x_{0}=1.2443 \times 10^{-3} \mathrm{~m}$. The experimentally measured force-gapcurrent relationship is shown on Fig. 5.

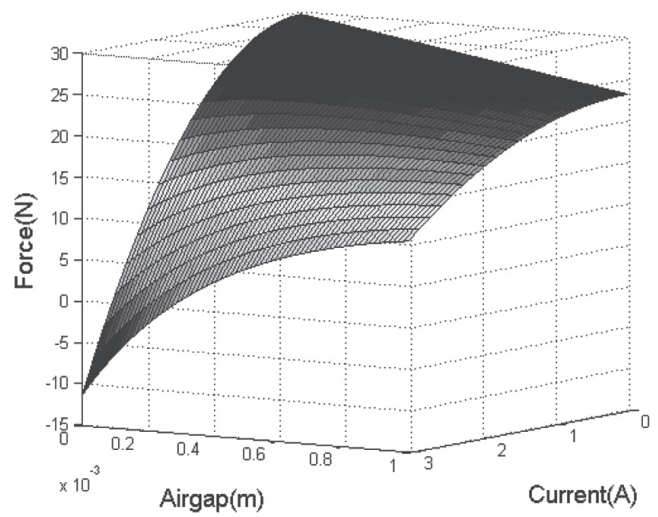

Fig. 5. Experimental Force-Gap-Current Relationship in the HMSA

\section{Experimental system modelling and control design}

Fig. 6 shows the block diagram of our one-DOF motion control system using HMSA. The position feedback signal is conditioned and used by a DSP board for control computation. The calculated control effort is fed to a servo amplifier to produce the desired current and drive the HMSA. The mass and stiffness of the beam is $M=1.2 \mathrm{~kg}$ and $K_{f}=2000 \mathrm{~N} / \mathrm{m}$ respectively. Assuming the initial gap is $g_{0}$ and the discrete state vector is $x(k)=\left[x_{1}(k)\right.$ $\left.x_{2}(k)\right]^{T}$ with sampling time $\Delta t$, the state space equations are:

$$
\begin{aligned}
& x_{1}(k+1)=x_{2}(k) \Delta t+x_{1}(k) \\
& x_{2}(k+1)=\frac{1}{M}\left(K_{1} x_{1}(k)+K_{2}-K_{3} \frac{I^{2}(k)}{\left(x_{1}(k)+x_{0}\right)^{2}}+K_{f}\left(\frac{g_{0}}{2}-x_{1}(k)\right)\right) \Delta t+x_{2}(k)
\end{aligned}
$$

Equation (3) can be converted to the controllability canonical form with bounded uncertainty: 


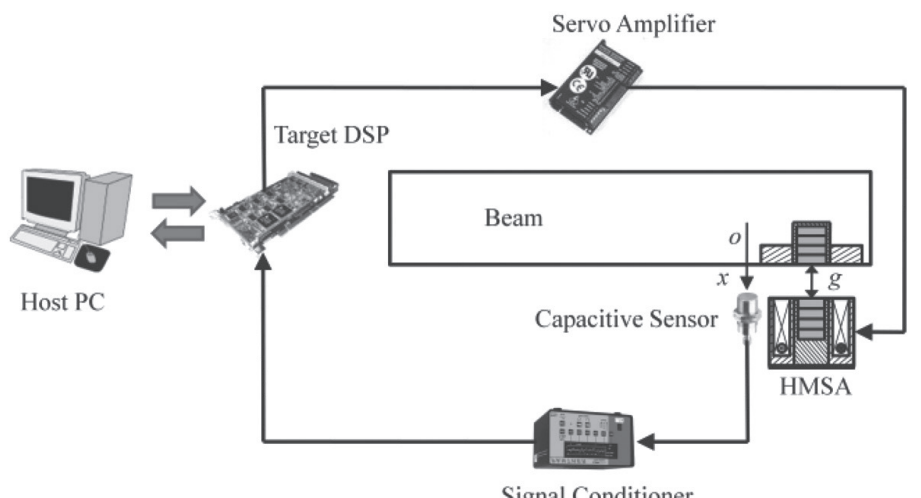

Signal Conditioner

Fig. 6. Experimental One DOF Motion Control System

$$
\begin{aligned}
& x_{1}(k+1)=x_{2}(k) \Delta t+x_{1}(k) \\
& x_{2}(k+1)=\left(f\left(x_{1}(k)\right)+g\left(x_{1}(k)\right) u(k)\right) \Delta t+x_{2}(k)
\end{aligned}
$$

Where

$$
\begin{aligned}
& u(k)=I^{2}(k) \\
& f\left(x_{1}(k)\right)=\left(K_{1}-K_{f}\right) x_{1}(k)+K_{2}+\frac{1}{2} K_{f} g_{0} \\
& g\left(x_{1}(k)\right)=-\frac{K_{3}}{\left(x_{1}(k)+x_{0}\right)^{2}}
\end{aligned}
$$

and $G_{1} \leq g\left(x_{1}(k)\right) \leq G_{2}<0$ is a bounded uncertain control gain. If the reference trajectory is $x_{r}(k)=\left[x_{1 r}(k) x_{2 r}(k)\right]^{T}$, and the error vector is $e(k)=\left[x_{1}(k)-x_{1 r}(k) x_{2}(k)-x_{2 r}(k)\right]^{T}$, a sliding surface useful for tracking can be written as:

$$
s(k)=c^{T} e(k) \text {, where } c=\left[\begin{array}{ll}
\lambda & 1
\end{array}\right]^{T} \text { and } \lambda>0 .
$$

Our goal is to find a digital control law $u(k)$ that can quickly drive the state trajectory to (5) and keep it there within a specified boundary $\{e(k) \| s(e)<\varepsilon, \varepsilon>0\}$. Instead of the conventional equivalent control approach, we propose an alternate method based on specifying a reaching law with linear rate:

$$
s(k+1)-s(k)=-q s(k) \Delta t-\operatorname{sgn}(s(k)) \varepsilon \Delta t, q>0
$$

From Equation (5):

$$
\begin{array}{r}
s(k+1)-s(k)=c^{T}(e(k+1)-e(k))=x_{2}(k+1)-x_{2}(k) \\
-\left(x_{2 r}(k+1)-x_{2 r}(k)\right)+\lambda\left(x_{1}(k+1)-x_{1}(k)-\left(x_{1 r}(k+1)-x_{1 r}(k)\right)\right)
\end{array}
$$

From Equation (4):

$$
x_{2}(k+1)-x_{2}(k)=\left(f\left(x_{1}(k)\right)+g\left(x_{1}(k)\right) u(k)\right) \Delta t
$$

Substituting (6) and (8) into (7), a quasi-sliding mode control law can be defined as: 


$$
u(k)=\frac{\widehat{u}(k)-s(k) q-\operatorname{sgn}(s(k)) \varepsilon}{g\left(x_{1}(k)\right)}
$$

where

$$
\begin{aligned}
\hat{u}(k)=- & f\left(x_{1}(k)\right)+\frac{1}{\Delta t}\left(x_{2 r}(k+1)-x_{2 r}(k)\right) \\
& -\frac{\lambda}{\Delta t}\left(x_{1}(k+1)-x_{1}(k)-\left(x_{1 r}(k+1)-x_{1 r}(k)\right)\right)
\end{aligned}
$$

To ensure finite reaching time, the following sliding condition must be satisfied:

$$
s(k+1)-s(k)<-\eta \operatorname{sgn}(s(k)) \Delta t, \eta>0
$$

Comparing (11) and (6) yields:

$$
\varepsilon \geq|\xi(D+\eta)-s(k) q|+(\xi-1)|\hat{u}(k)|
$$

where $\xi=\left(G_{2} / G_{1}\right)^{1 / 2}$ is the gain margin (Slotine,1987). It has been demonstrated in (Gao, 1993) that the time required to reach the sliding surface from an arbitrary initial state is given by:

$$
T=\frac{1}{q} \ln \left(1+\frac{q|s|}{\varepsilon}\right)
$$

which shows that for a given value of $q$ the reaching time is reduced by increasing $\varepsilon$. However, a higher value of $\varepsilon$ also leads to more chatter, as can be seen from the control law (9). To reduce chatter, we replace the switching function in (6), (9) and (11) with a saturation function, and introduce a boundary layer $\phi$. The quasi-sliding control law is finally given by:

$$
u(k)=\frac{\widehat{u}(k)-s(k) q-\operatorname{sat}\left(\frac{s(k)}{\phi}\right) \varepsilon}{g\left(x_{1}(k)\right)}
$$

Both (9) and (14) show that full state feedback is required to calculate the control action. In a practical real-time implementation of the HMSA, only position feedback would be available. A high-gain state observer is used to estimate velocity from position measurements.

Given the nominal model $\hat{\phi}\left(\hat{x}_{1}(k), u(k)\right)=f\left(\hat{x}_{1}(k)\right)+g\left(\hat{x}_{1}(k)\right) u(k)$, the perturbations on $\hat{\phi}$ can be modelled as $\phi_{d}\left(\hat{x}_{1}(k), x_{1}(k)\right)$ so that

$$
\phi\left(x_{1}(k), u(k)\right)=\hat{\phi}\left(\hat{x}_{1}(k), u(k)\right)+\phi_{d}\left(\hat{x}_{1}(k), x_{1}(k)\right) .
$$

As a result, (8) can be rewritten as:

$$
\begin{aligned}
& x_{1}(k+1)=x_{2}(k) \Delta t+x_{1}(k) \\
& x_{2}(k+1)=\phi\left(x_{1}(k), u(k)\right) \Delta t+x_{2}(k) \\
& y(k)=x_{1}(k)
\end{aligned}
$$

where $\hat{x}_{1}(k)$ is the estimated position and $y(k)$ is the measured position. A velocity observer for (19) can be given by: 


$$
\begin{aligned}
& \hat{x}_{1}(k+1)=\left(\hat{x}_{2}(k)+h_{1}\left(y-\hat{x}_{1}(k)\right)\right) \Delta t+\hat{x}_{1}(k) \\
& \hat{x}_{2}(k+1)=\left(\hat{\phi}\left(\hat{x}_{1}(k), u(k)\right)+h_{2}\left(y-\hat{x}_{1}(k)\right)\right) \Delta t+\hat{x}_{2}(k) \\
& \hat{v}(k)=\hat{x}_{2}(k)
\end{aligned}
$$

where $\hat{v}(k)$ is the estimated velocity, $\mathrm{h}_{1}$ and $\mathrm{h}_{2}$ are observer gains. Subtracting (16) from (15) shows the dynamics of the estimation error:

$$
\begin{aligned}
& \tilde{x}_{1}(k+1)=-\left(h_{1} \tilde{x}_{1}(k)+\tilde{x}_{2}(k)\right) \Delta t+\tilde{x}_{1}(k) \\
& \tilde{x}_{2}(k+1)=-\left(h_{2} \tilde{x}_{1}(k)+\phi_{d}\left(\tilde{x}_{1}(k), x_{1}(k)\right)\right) \Delta t+\tilde{x}_{2}(k)
\end{aligned}
$$

A block diagram of the proposed observer-based quasi-sliding mode control is shown on Fig. 7.

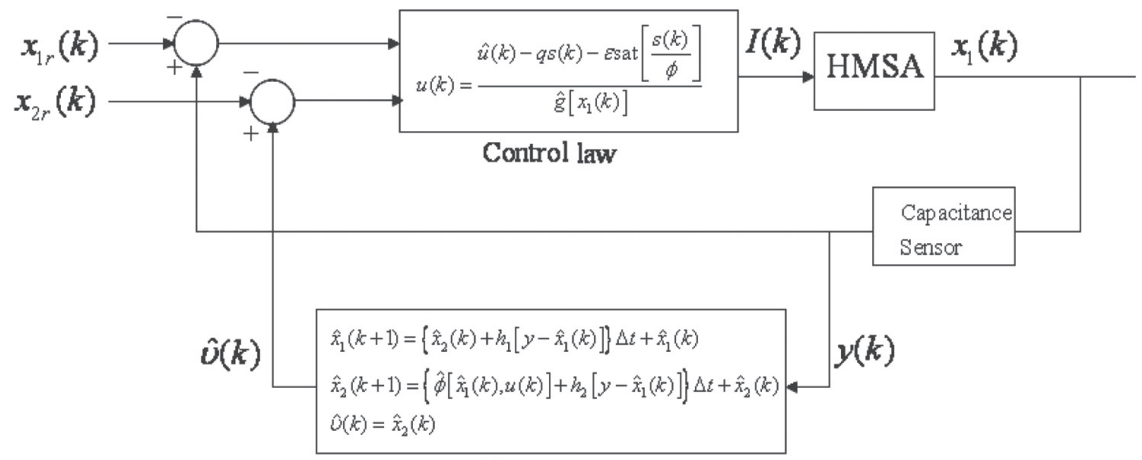

Observer

Fig. 7. Observer based Quasi Sliding Mode Control of the proposed HMSA

\section{Experimental results}

Several experiments were carried out to test both positioning and tracking performances of the proposed controller. All control algorithms were implemented on a TI TMS320C67 DSP computer, at a sampling rate of $2 \mathrm{kHz}$.

\subsection{Consecutive step response}

To demonstrate the positioning capabilities of the proposed HMSA, 10 $\mu \mathrm{m}$ consecutive step commands are used. The target is stabilized at its initial gap of $1190 \mu \mathrm{m}$ and then multiple step commands are executed. To obtain large gap measurement capability, the capacitive sensor is set at coarse measurement mode. Fig. 8 shows the experimental positioning performance of the proposed quasi-sliding control with linear reaching rate. Fig. 9 shows the experimental result of the conventional PID control with feedback linearization. The performances of both controllers in terms of the overshoot, settling time and the steady state error are listed in Table 2. 

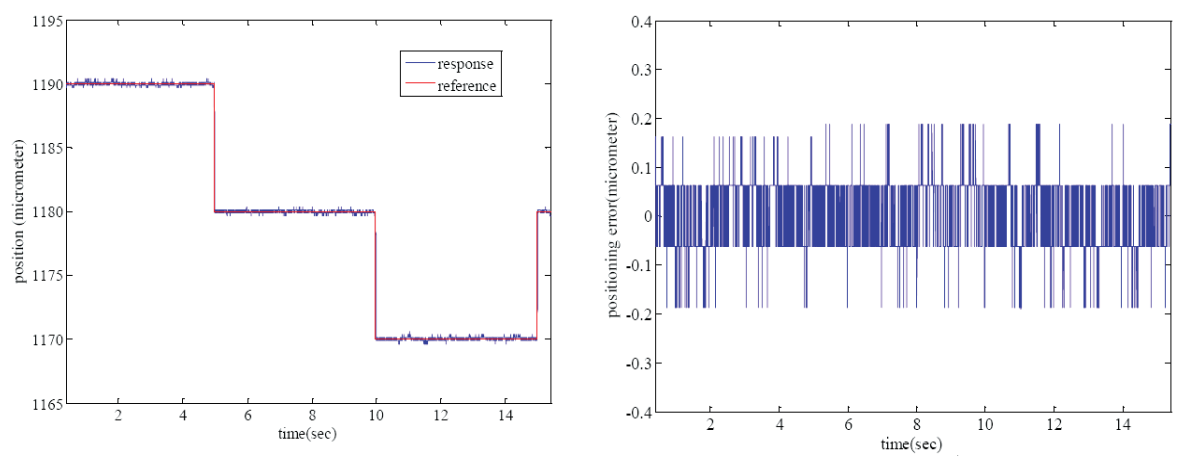

Fig. 8. Positioning Performances of Quai Sliding-Mode Control with Linear Reaching Law (Left) $10 \mu \mathrm{m}$ consecutive step response (Right) positioning error
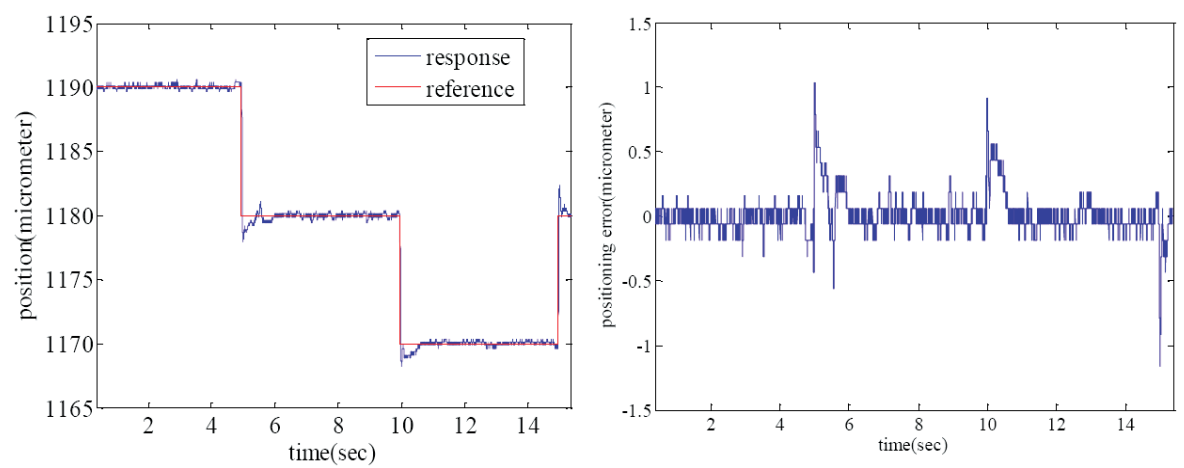

Fig. 9. Positioning Performances of PID Control with feedback linearization (Left) $10 \mu \mathrm{m}$ consecutive step response (Right) positioning error

\begin{tabular}{|c|c|c|}
\hline Controllers & $\begin{array}{c}\text { Quasi-sliding mode control } \\
\text { with linear reaching law }\end{array}$ & $\begin{array}{c}\text { PID with feedback } \\
\text { linearization }\end{array}$ \\
\hline overshoot & none & $1 \mu \mathrm{m}$ \\
\hline settling time & $0.02 \mathrm{~s}$ & $0.7 \mathrm{~s}$ \\
\hline steady state error & $\pm 200 \mathrm{~nm}$ & $\pm 200 \mathrm{~nm}$ \\
\hline
\end{tabular}

Table 2. Performances for $10 \mu \mathrm{m}$ consecutive step responses 


\subsection{Tracking response}

To test the tracking performance of the proposed HMSA actuator, it is commanded to follow a trapezoidal trajectory starting from $1000 \mu \mathrm{m}$ with amplitude of $400 \mathrm{~nm}$. The sliding control law (14) is implemented and the results are shown on Fig. 10. To exhibit more precise motion control capability, the capacitive sensor is set at fine measurement mode which gives tracking error of $\pm 60 \mathrm{~nm}$.
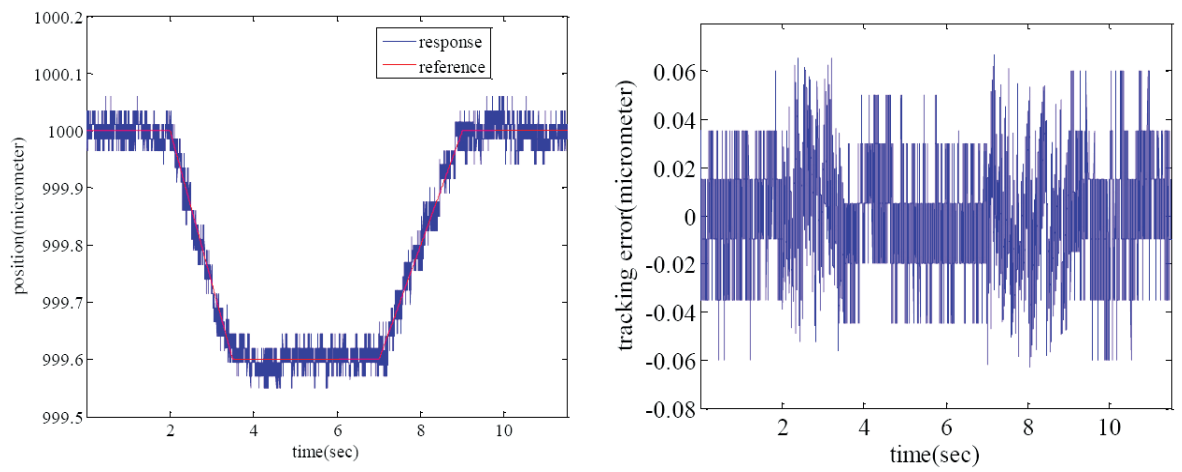

Fig. 10. Tracking Performances of Quasi Sliding-Mode Control with Linear Reaching Law (Left) $400 \mathrm{~nm}$ trapezoidal tracking (Right) tracking error

\section{Conclusion}

A novel 1-DOF hybrid magnetic suspension actuator that has a great promise to realize large gap while avoiding large levitation currents and actuator saturation has been presented. In the proposed design, a pair of permanent magnets in repulsive configuration is located coaxial with an attractive force electromagnet. Controlled attractive forces are used to provide stabilization. The passive push-active pull strategy offers substantial advantages over other designs described in the literature when a large load is to be accurately suspended over a large range of travel. The proposed actuator can be used modularly to control multiple axes of motion in a multi-DOF positioning application that requires millimeter-range travel with fast response and sub-micron accuracy.

The force model parameters of the proposed actuator were identified using a multidimensional unconstrained nonlinear optimization method. To control the designed magnetic suspension system, a quasi-sliding mode controller (QSM) has been presented, in which a linear reaching law is used instead of the typical Lyapunov function approach. Since full knowledge of the state vector is required, a nonlinear high-gain observer was also designed and implemented. For comparison, a PID control with standard feedback linearization is also implemented for the magnetic suspension system. Several experiments were performed to demonstrate both the positioning and tracking capabilities of the proposed actuator. A fast, stable response with low chatter has been achieved via QSM. In a 
positioning control, the proposed method demonstrates faster response and better transient performance when compared with those of standard feedback linearization. In a tracking control, it also achieves a minimal positioning error of $\pm 60 \mathrm{~nm}$ which is very useful for systems in which steady-state accuracy is critical.

\section{References}

Chikazumi, S. (2005). Physics of Ferromagnetism. Oxford University Press, pp. 19-21.

Craig, D. and Khamesee, M. B. (2007). Motion Control of a Large Gap Magnetic Suspension System for Microrobotic Manipulation. J. Phys. D: Appl. Phys. 40, pp. 3277-3285.

Gao, W., and Hung, J. C. (1993). Variable Structure Control of Nonlinear Systems: A New Approach. IEEE Trans. Industrial Electronics, vol. 40, No.1, pp.45-55.

Gutierrez, H. M. and Ro, P. I. (2005). Magnetic Servo Levitation by Sliding-Mode Control of Nonaffine Systems with Algebraic Input Invertibility. IEEE Trans. Industrial Electronics, vol. 52, No.5, pp.1449-1455.

Hung, J. Y., Gao, W., and Hung, J. C. (1993). Variable Structure Control: A Survey. IEEE Trans. Industrial Electronics, vol. 40, No.1, pp.2-22.

Khamesee, M. B. and Shameli, E. (2005). Regulation Technique for a large gap magnetic field for 3D non-contact manipulation. The Elsevier Journal of Mechatronics, 15, pp. 10731087.

Kuo, S-K, Shan, X., and Menq, C-H. (2003). Large Travel Ultra Precision Motion Control of a Magnetic-Suspension Stage. IEEE Trans. Mechatronics, vol. 8, No.3, pp.334-341.

Lin, C. E. and Jou, H. L.. (1993). Force Model Identification for Magnetic Suspension Systems via Magnetic Field Measurement. IEEE Trans. Instrumentation and Measurement, vol. 42, No.3, pp.767-771.

Lin, F-J, Teng, L-T and Shieh, P-H. (2007). Intelligent Sliding-Mode Control Using RBFN for Magnetic Levitation System. IEEE Trans. Industrial Electronics, vol. 54, No.3, pp.1752-1762.

Ludwick, S. J., Trumper, D.L., and Holmes, M. L.. (1996). Modeling and Control of a Six Degree-of-Freedom Magnetic/Fluidic Motion Control Stage. IEEE Trans. Control Systems Technology, vol. 4, No.5, pp.553-564, September.

Mittal, S. and Menq, C-H. (1997). Precision Motion Control of a Magnetic Suspension Actuator Using a Robust Nonlinear Compensation Scheme. IEEE Trans. Mechatronics, vol. 2, No.4, pp.268-280.

Oliveira, V. A., Costa, E. F., and Vargas, J. B. (1999). Digital Implementation of a Magnetic Suspension Control System for Laboratory Experiments. IEEE Trans. Education, vol. 42, No.4, pp.315-322.

Robbins, W. P., Polla, D. L., and Glumac, D. E. (1991). High-Displacement Piezoelectric Actuator Utilizing a Meander-Line Geometry-Part I: Experimental Characterization. IEEE Trans. Ultrasonics, Ferroelectrics, and Frequency Control, vol. 38, No.5, pp.454-460.

Slotine, J.J. and Li, W. (1987). Applied Nonlinear Control. Englewood Cliffs, NJ Prentice Hall. 
Trumper D. L., Olson, S. M., and Subrahmanyan, P. K.. (1997). Linearizing Control of Magnetic Suspension Systems. IEEE Trans. Control Systems Technology, vol. 5, No.4, pp.427-438. 
(c) 2010 The Author(s). Licensee IntechOpen. This chapter is distributed under the terms of the Creative Commons AttributionNonCommercial-ShareAlike-3.0 License, which permits use, distribution and reproduction for non-commercial purposes, provided the original is properly cited and derivative works building on this content are distributed under the same license. 